PROCEEDINGS OF THE

AMERICAN MATHEMATICAL SOCIETY

Volume 136, Number 12, December 2008, Pages 4365-4372

S 0002-9939(08)09452-5

Article electronically published on June 27, 2008

\title{
A MARKOV DILATION FOR SELF-ADJOINT SCHUR MULTIPLIERS
}

\author{
ÉRIC RICARD
}

(Communicated by Marius Junge)

\begin{abstract}
We give a formula for Markov dilation in the sense of Anantharaman-Delaroche for real positive Schur multipliers on $\mathbb{B}(H)$.
\end{abstract}

The classical theory of semigroups has many applications and connections with ergodic theory, martingales and probability (see [12]). The recent developments of noncommutative integration in von Neumann provide analogues of these notions ([1], [5], 6]). For instance, classical Markov semigroups on a probability space are generalized to semigroups of unital completely positive maps preserving a given faithful state. It is natural to try to adapt techniques from the commutative theory to the noncommutative one. Dealing with $C^{*}$-algebras, Sauvageot [1] has given a construction of a Markov $C^{*}$-dilation for a semigroup in the spirit of DaniellKolmogorov. One of the main tools in the classical setting is Rota's dilation theorem [10, [12. It states that any Markovian map (unital, positive, self-adjoint on $L_{2}$ and contractive on all $L_{p}$ 's) has a nice dilation in terms of a reversed martingale, namely $Q^{2 n}=\hat{E} \circ E_{n}$, where the $E_{n}$ are conditional expectations from a decreasing filtration and $\hat{E}$ is another conditional expectation. This is closely related to the construction of Markov chains. Anantharaman-Delaroche states a counterpart of it for von Neumann algebras in [1]. Unfortunately some extra technical condition is needed. She called it "factorization" or Markov dilation. It is unknown if this condition holds for any Markov operator. The aim of this paper is to discuss this factorization for some concrete and basic examples.

Let's start with Stinespring's dilation theorem for $C^{*}$-algebras. It asserts that given a unital completely positive map $u: A \rightarrow \mathbb{B}(H)$, one can find a Hilbert space $K$ containing $H$, a representation $\pi$ from $A$ to $\mathbb{B}(K)$ so that $u$ is just the composition of $\pi$ and the natural conditional expectation onto $\mathbb{B}(H)$. More precisely, $K$ is the Hilbert space $A \otimes_{u} H$ obtained by completion of $A \otimes H$ for the scalar product:

$$
\forall a, a^{\prime} \in A, h, h^{\prime} \in H \quad\left\langle a \otimes h, a^{\prime} \otimes h^{\prime}\right\rangle_{K}=\left\langle h, u\left(a^{*} a^{\prime}\right) \cdot h^{\prime}\right\rangle_{H} .
$$

Received by the editors July 23, 2007, and, in revised form, November 12, 2007.

2000 Mathematics Subject Classification. Primary 46L53.

Key words and phrases. Completely positive maps, Schur multipliers.

The author is supported by research program ANR-06-BLAN-0015.

(C)2008 American Mathematical Society Reverts to public domain 28 years from publication 
The inclusion map $\pi: A \rightarrow \mathbb{B}(K)$ is given by $\pi(a)\left(a^{\prime} \otimes h\right)=a a^{\prime} \otimes h$. The embedding from $H$ into $K$ is $h \mapsto 1 \otimes h$. The formula for the projection $P$ from $K$ onto $H$ is $P(a \otimes h)=1 \otimes u(a) . h$, and one has

$$
u(a)=P \pi(a)_{\mid H} .
$$

This result is used as the very basic step in Sauvageot's construction. Its main drawback is that when dealing with von Neumann algebras (with faithful state or trace) for applications in $L_{p}$ spaces (see [1], [5], 6]), one would like to have a dilation that stays in this category. Therefore Claire Anantharaman-Delaroche introduces the notion of factorizable maps in [1, which we describe precisely now. We will use classical notation for von Neumann algebras as in [13, 1].

Let $M$ and $N$ be von Neumann algebras with normal faithful states $\phi$ and $\psi$.

Definition. A $(\phi, \psi)$-Markov operator $u:(M, \phi) \rightarrow(N, \psi)$ is a normal unital completely positive map so that $\psi \circ u=\phi$ and that intertwines the modular groups of $\phi$ and $\psi\left(u \circ \sigma_{t}^{\phi}=\sigma_{t}^{\psi} \circ u\right)$.

One says that $u$ admits a factorization if there exist another von Neumann algebra $\tilde{M}$ with a faithful state $\tilde{\phi}$ and normal representations $\pi: M \rightarrow \tilde{M}, \rho: N \rightarrow$ $\tilde{M}$ that are $(\phi, \tilde{\phi})$ - and $(\psi, \tilde{\phi})$-Markov maps, with

$$
\psi(u(m) n)=\tilde{\phi}(\pi(m) \rho(n)) .
$$

We say that $(\tilde{M}, \tilde{\phi})$ is a Markov dilation for $u$. The conditions on modular groups imply that there is actually a $(\tilde{\phi}, \psi)$-Markov conditional expectation $\mathbb{E}: \tilde{M} \rightarrow N$ and $u=\mathbb{E} \circ \pi$.

A natural question is which maps are factorizable. The aim of this paper is to give a positive answer for real multipliers.

Viewing $N$ acting as a subalgebra of $\mathbb{B}\left(L_{2}(N, \phi)\right)$, one can notice that if $u$ is factorizable, then $M \otimes_{u} L_{2}(N, \phi)$ consists exactly in the norm closure of $\pi(M) . \rho(N)$ in $L_{2}(\tilde{M}, \tilde{\phi})$.

In the commutative setting, Stinespring's dilation is actually a Markov dilation. This follows from the fact that $N$ acts on $M \otimes_{u} L_{2}(N, \phi)$ by right multiplications. The commutative von Neumann algebra generated by $M$ and $N$ in $\mathbb{B}\left(M \otimes_{u} L_{2}(N, \phi)\right)$ is $\tilde{M}$ and the state is $\tilde{\phi}(x)=\langle 1 \otimes 1, x .(1 \otimes 1)\rangle$. This is the classical Markov construction.

In the sequel, we are only interested in the case $M=N$ and $\phi=\psi$, and we keep the above notation.

We start with some remarks on the set of factorizable maps.

Any state-preserving homomorphism $\alpha: M \rightarrow M$ is factorizable; a dilation is obtained with $\tilde{M}=M, \tilde{\phi}=\phi, \pi=\alpha$ and $\rho=\mathrm{Id}$.

Any Markovian conditional expectation $\mathbb{E}: M \rightarrow M$ onto a subalgebra $N$ is factorizable. A dilation is given by the free product with amalgamation over $N$ : $(\tilde{M}, \tilde{\phi})=(M, \phi) *_{N}(M, \phi), \pi$ is the homomorphism onto the first copy of $M$ in $\tilde{M}$ and $\rho$ onto the second one. We refer to [14, [9] for definitions. In particular, taking $N=\mathbb{C} .1, \phi$ is factorizable.

The dilation is nonunique in general. For instance, $\phi$ can also be dilated in $(M, \phi) \bar{\otimes}_{\min }(M, \phi)$ with the obvious inclusions.

The fixed point algebra $N$ by a $\phi$-Markov map plays a particular role in the dilation, as we must have $\pi(n)=\rho(n)$ for any $n \in N$. 
If $T$ is $\phi$-Markovian, then it is known that the adjoint of $T$ on $L^{2}(M, \phi)$ comes also from a $\phi$-Markovian map denoted by $T^{\star}$ (see [1]), that is,

$$
\phi(x T(y))=\phi\left(T^{\star}(x) y\right) .
$$

We say that $T$ is self-adjoint if $T^{\star}=T$.

Proposition. The set of factorizable $\phi$-Markovian operators on $M$ is convex, stable by composition, by the involution * and closed for the point weak-* topology.

Proof. About the involution, it suffices to exchange the roles of $\pi$ and $\rho$ as for analytic elements

$$
\begin{aligned}
\phi\left(T^{\star}(y) x\right) & =\phi\left(T\left(\sigma_{i}^{\phi}(x)\right) y\right)=\tilde{\phi}\left(\pi\left(\sigma_{i}^{\phi}(x)\right) \rho(y)\right) \\
& =\tilde{\phi}\left(\sigma_{i}^{\tilde{\phi}}(\pi(x)) \rho(y)\right)=\tilde{\phi}(\rho(y) \pi(x)) .
\end{aligned}
$$

Let $u_{i}$ be $\phi$-Markovian on $M$ with dilation $\left(\tilde{M}_{i}, \tilde{\phi}_{i}\right)$ and morphisms $\pi_{i}$ and $\rho_{i}$.

A dilation for $\lambda u_{1}+(1-\lambda) u_{2}$ is given by

$$
\left(\tilde{M}_{1} \oplus \tilde{M}_{1}, \lambda \tilde{\phi}_{1} \oplus(1-\lambda) \tilde{\phi}_{2}\right)
$$

with morphisms $M \rightarrow \tilde{M}_{1} \oplus \tilde{M}_{2}, \pi_{1} \oplus \pi_{2}$ and $\rho_{1} \oplus \rho_{2}$.

A dilation for $u_{2} \circ u_{1}$ comes from the free product construction. Consider $(\tilde{M}, \tilde{\phi})$ given by $\left(\tilde{M}_{1}, \tilde{\phi}_{1}\right) *_{M}\left(\tilde{M}_{2}, \tilde{\phi}_{2}\right)$, where the amalgamation is taken over the copy of $M$ coming from $\rho_{1}$ in $M_{1}$ and $\pi_{2}$ in $M_{2}$ (note that there are indeed conditional expectations onto them). Let $\mathbb{E}$ be the conditional expectation onto the amalgamated copy of $M$. From the definition of a dilation, we get that $\mathbb{E}\left(\pi_{1}(x)\right)=\rho_{1}\left(u_{1}(x)\right)$ and $\mathbb{E}\left(\rho_{2}(y)\right)=\pi_{2}\left(u_{2}^{\star}(y)\right)$ for $x, y \in M$. A classical computation in free products gives

$$
\begin{aligned}
\tilde{\phi}\left(\pi_{1}(x) \rho_{2}(y)\right) & =\tilde{\phi}\left(\mathbb{E}\left(\pi_{1}(x)\right) \mathbb{E}\left(\rho_{2}(y)\right)\right)=\tilde{\phi}\left(\rho_{1}\left(u_{1}(x)\right) \pi_{2}\left(u_{2}^{\star}(y)\right)\right) \\
& =\tilde{\phi}\left(\rho_{1}\left(u_{1}(x) u_{2}^{\star}(y)\right)\right)=\phi\left(\left(u_{2} \circ u_{1}\right)(x) . y\right) .
\end{aligned}
$$

The statement about the closure property is obtained by taking an ultraproduct (see [8]) and cutting with some projections to make representations normal and the state faithful. Technical details can be found in [4.

Among other permanence properties, it was observed in [5] that the free product of factorizable maps is still factorizable and the dilation is simply the free product of the dilations.

As a corollary of Proposition 2, it was pointed out to us by Claire AnantharamanDelaroche that any tracial Markov map on $\mathbb{M}_{2}$ is factorizable as the extreme points of such maps are exactly the automorphisms.

We now come to Schur multipliers on $\mathbb{B}\left(\ell_{2}^{I}\right)$ with canonical orthonormal basis $\left(e_{i}\right)_{i \in I}$. We will assume that the state $\phi$ has a diagonal density $D=\sum \lambda_{i} e_{i} \otimes e_{i}$ for the canonical basis with respect to the trace. We have $\lambda_{i}>0$ and $\sum \lambda_{i}=1$. The modular group of $\phi$ is $\sigma_{t}^{\phi}(x)=D^{-i t} x D^{i t}$.

We represent elements in $\mathbb{B}\left(\ell_{2}^{I}\right)$ as matrices $\mathbb{M}_{I}$ of size $I$. Given any matrix $T=\left(t_{i, j}\right) \in \mathbb{M}_{I}$, we say that $T$ is a Schur multiplier if the following map is well defined:

$$
M_{T}=\left\{\begin{array}{llc}
\mathbb{B}\left(\ell_{2}^{I}\right) & \rightarrow & \mathbb{B}\left(\ell_{2}^{I}\right) \\
\left(x_{i, j}\right) & \mapsto & \left(t_{i, j} x_{i, j}\right) .
\end{array}\right.
$$

A characterization of bounded multipliers can be found in [7]. A multiplier is (completely) positive if and only if its symbol $T$ is positive in the sense that for any 
finite set $F \subset I,\left(t_{i, j}\right)_{i, j \in F}$ is positive in $\mathbb{M}_{F} . M_{T}$ is unital if $t_{i, i}=1$ for all $i \in I$. $M_{T}$ is normal unital and completely positive iff there exist norm 1 vectors $x_{i} \in \ell_{2}^{I}$ so that $t_{i, j}=\left\langle x_{i}, x_{j}\right\rangle$.

From these observations, it is clear that any unital completely positive Schur multiplier is $\phi$-Markovian.

In the opposite way, if a map $u$ commutes with the modular group of $\phi$ and $\left(\log \left(\lambda_{i}\right)\right)_{i \in I}$ is independent over $\mathbb{Q}$ in $\mathbb{R}$, then $u$ has to be a Schur multiplier.

The adjoint of $M_{T}$ is $M_{T^{t}}$ where $T^{t}=\left(t_{j, i}\right)_{i, j}$. So any self-adjoint $\phi$-Markovian Schur multiplier has to have real coefficients.

Theorem. Any positive self-adjoint $\phi$-Markovian Schur multiplier is factorizable.

To construct the dilation, we will need the fermion algebras. Let $K$ be a real Hilbert space with complexification $K_{\mathbb{C}}$. We briefly recall their construction and the more general $q$-deformed algebras in the spirit of $[2]$. The $q$-Fock $(-1 \leqslant q<1)$ space over $K$ is

$$
\mathcal{F}_{q}(K)=\mathbb{C} \Omega \oplus \bigoplus_{k} K_{\mathbb{C}}^{\otimes_{q} k}
$$

where the scalar product on $K_{\mathbb{C}}^{\otimes_{q} n}$ is given by

$$
\left\langle k_{1} \otimes \ldots \otimes k_{n}, h_{1} \otimes \ldots \otimes h_{n}\right\rangle_{q}=\sum_{\sigma \in S_{n}} q^{|\sigma|}\left\langle k_{i}, h_{\sigma_{i}}\right\rangle_{K_{\mathbb{C}}}
$$

and where $S_{n}$ is the symmetric group and $|\sigma|$ the number of inversions of the permutation $\sigma$. When $q=-1$, this is just the antisymmetric tensor product $K_{\mathbb{C}}^{\wedge n}$.

The creation operator for $e \in K$ is given by

$$
l(e) .\left(h_{1} \otimes \ldots \otimes h_{n}\right)=e \otimes h_{1} \otimes \ldots \otimes h_{n} .
$$

They satisfy the $q$-relation

$$
l(f)^{*} l(e)-q l(e) l(f)^{*}=\langle f, e\rangle_{K} I d .
$$

The $q$-von Neumann algebra is

$$
\Gamma_{q}(K)=\left\{\omega(e)=l(e)+l(e)^{*} ; e \in K\right\}^{\prime \prime} .
$$

It is type $I I_{1}$ with trace $\tau(x)=\langle\Omega, x . \Omega\rangle_{\mathcal{F}_{q}(K)}$.

We are mainly concerned with the fermion algebra when $q=-1$. If $e \in K$ has norm 1 , then $\omega(e)$ is a symmetry, i.e. self-adjoint with $\omega(e)^{2}=1$. Moreover we have

$$
\forall e, f \in K \quad \tau(\omega(e) \omega(f))=\langle e, f\rangle_{K} .
$$

Proof. We will use the notation $e_{i, j}$ for the canonical basis of $\mathbb{B}\left(\ell_{2}^{I}\right)$.

Let $M_{T}$ be a self-adjoint Markovian Schur multiplier. As $t_{i, j}=\left\langle x_{i}, x_{j}\right\rangle$ is real, $T$ defines a new scalar product on the real linear span of $e_{i}$ 's by the formula

$$
\left\langle\sum a_{i} e_{i}, \sum b_{i} e_{i}\right\rangle_{T}=\left\langle\sum a_{i} x_{i}, \sum b_{i} x_{i}\right\rangle_{\ell_{2}^{I}}=\sum_{i, j} a_{i} b_{j} t_{i, j} .
$$

We call $\ell_{2, T}$ the real Hilbert space obtained after quotient and completion. We still denote by $e_{i}$ the representative of $e_{i}$ in $\ell_{2, T}$. We have

$$
\left\langle e_{i}, e_{j}\right\rangle_{T}=t_{i, j}=t_{j, i} .
$$


Let $\tilde{M}=\mathbb{B}\left(\ell_{2}^{I}\right) \bar{\otimes}_{\min } \Gamma_{-1}\left(\ell_{2, T}\right)$ with normal faithful state $\tilde{\phi}=\phi \otimes \tau$. Let

$$
d=\sum_{i} e_{i, i} \otimes \omega\left(e_{i}\right) \in \tilde{M}
$$

It is a unitary (symmetry) in the centralizer of $\tilde{\phi}$, as $e_{i, i}$ are in the centralizer of $\phi$.

For $x \in \tilde{M}$, define

$$
\mathcal{U}(x)=d x d .
$$

This is a $\tilde{\phi}$-Markovian map and a representation as $d^{2}=1$.

Let $\pi: M \rightarrow \tilde{M}$ be the obvious inclusion Id $\otimes 1$. Define $\rho: M \rightarrow \tilde{M}$ as $\rho=\mathcal{U} \circ \pi$. It is clear that $\pi$ and $\rho$ are $(\phi, \tilde{\phi})$-Markovian and for $x=\left(x_{i, j}\right)$ and $y=\left(y_{i, j}\right)$ finite matrices:

$$
\begin{aligned}
\tilde{\phi}(\pi(x) \rho(y)) & =\phi \otimes \tau((x \otimes 1) d(y \otimes 1) d) \\
& =\phi \otimes \tau\left(\left(x_{i, j} \cdot 1\right) \cdot\left(y_{i, j} \omega\left(e_{i}\right) \omega\left(e_{j}\right)\right)\right) \\
& =\sum_{i, j} \lambda_{i} x_{i, j} y_{j, i} \tau\left(\omega\left(e_{i}\right) \omega\left(e_{j}\right)\right) \\
& =\sum_{i, j} \lambda_{i} x_{i, j} y_{j, i} t_{i, j} \\
& =\phi(T(x) y) .
\end{aligned}
$$

When $T=\mathrm{Id}, M_{T}$ is a conditional expectation, and this dilation is very different from the one obtained by free product.

Combining the previous example with the permanence properties gives a wide class of factorizable maps. If $I$ is finite and $\phi$ is the trace, we can take compositions of multipliers in different bases (and with conditional expectations, representations) and convex combinations of them. We do not know whether we can achieve all tracial Markovian maps for $\mathbb{M}_{n}$. It follows from Grothendieck's theorem that any completely bounded Schur multiplier is a multiple (less than the Grothendieck constant) of a convex combination of rank one multipliers (see [7]). In terms of Markov maps, any Markov Schur multiplier can be obtained as a linear combination of representations. Unfortunately it cannot be a convex one (or the Grothendieck constant would have to be exactly 1 ).

If one looks carefully, the von Neumann algebra generated by $\pi\left(\mathbb{B}\left(\ell_{2}^{I}\right)\right)$ and $\rho\left(\mathbb{B}\left(\ell_{2}^{I}\right)\right)$ is exactly $\mathbb{B}\left(\ell_{2}^{I}\right) \bar{\otimes} \Gamma_{-1}^{e}\left(\ell_{2, T}\right)$, where $\Gamma_{-1}^{e}\left(\ell_{2, T}\right)$ is the subalgebra of $\Gamma_{-1}\left(\ell_{2, T}\right)$ generated by even elements of the form $\omega\left(e_{i}\right) \omega\left(e_{j}\right)$.

Now that we have a dilation for Schur multipliers, thanks to the construction in [1, chapter 6], we have a noncommutative Markov chain. In our concrete setting, one can avoid this abstract construction (and free products). We follow the notation of [1] and classical notation for infinite tensor products (we drop the completion symbol). Let

$$
M=\mathbb{B}\left(\ell_{2}^{I}\right) \otimes \Gamma_{-1}^{e}\left(\ell_{2, T}\right)^{\otimes \infty} \subset \Gamma_{-1}\left(\ell_{2, T}\right)^{\otimes \infty}
$$

be equipped with the tensor product state. Actually, because of the commutation relations,

$$
\Gamma_{-1}^{e}\left(\ell_{2, T}\right)^{\otimes \infty} \subset \Gamma_{-1}\left(\ell_{2, T} \otimes \ell_{2}\right) .
$$

Let $J_{0}: \mathbb{B}\left(\ell_{2}^{T}\right) \rightarrow M$ be the natural inclusion given by

$$
J_{0}(x)=x \otimes 1 \otimes 1 \otimes \ldots .
$$


The letter $S$ stands for the shift on $\Gamma_{-1}^{e}\left(\ell_{2, T}\right)^{\otimes \infty}$ :

$$
S\left(x_{1} \otimes \ldots \otimes x_{n} \otimes 1 \otimes \ldots\right)=1 \otimes x_{1} \otimes \ldots \otimes x_{n} \otimes 1 \otimes \ldots .
$$

We will also need the symmetry

$$
d_{1}=\sum_{i} e_{i, i} \otimes \omega\left(e_{i}\right) \otimes 1 \otimes \ldots \in \mathbb{B}\left(\ell_{2}^{I}\right) \bar{\otimes} \Gamma_{-1}\left(\ell_{2, T}\right)^{\infty} .
$$

We have an injective morphism $\beta: M \rightarrow M$ given by

$$
\beta(x)=d_{1} S(x) d_{1} .
$$

If $J_{q}=\beta^{q} \circ J_{0}$, the $q^{t h}$ copy of $\mathbb{B}\left(\ell_{2}^{I}\right)$ is

$$
J_{q}\left(e_{i, j}\right)=e_{i, j} \otimes \underbrace{\omega\left(e_{i}\right) \omega\left(e_{j}\right) \otimes \ldots \otimes \omega\left(e_{i}\right) \omega\left(e_{j}\right)}_{q \text { times }} \otimes 1 \otimes \ldots
$$

The algebra $\mathcal{B}_{n]}$ generated by the first $n$ copies of $\mathbb{B}\left(\ell_{2}^{I}\right)$ is exactly

$$
\mathcal{B}_{n]}=\mathbb{B}\left(\ell_{2}^{I}\right) \otimes \Gamma_{-1}^{e}\left(\ell_{2, T}\right)^{\otimes n} \otimes \mathbb{C}^{\otimes \infty},
$$

and $\mathcal{B}_{[n}$, generated by $J_{q}\left(\mathbb{B}\left(\ell_{2}^{I}\right)\right)$ with $q \geqslant n$, is

$$
\mathcal{B}_{[n}=J_{n}\left(\mathbb{B}\left(\ell_{2}^{I}\right)\right) \cdot\left(\mathbb{C}^{\otimes n} \otimes \Gamma_{-1}^{e}\left(\ell_{2, T}\right)^{\otimes \infty}\right) .
$$

All maps preserve the involved modular groups, and if $\mathbb{E}_{n]}$ and $\mathbb{E}_{[n}$ are the conditional expectations onto $\mathcal{B}_{n]}$ and $\mathcal{B}_{[n}$, one can check the Markov properties

$$
\begin{aligned}
\mathbb{E}_{n]} & \circ J_{q}=J_{n} \circ T^{q-n}, \quad q \geqslant n, \\
\mathbb{E}_{n+q]} \circ \beta^{q} & =\beta^{q} \circ \mathbb{E}_{n]}, \\
\mathbb{E}_{[n} \circ J_{0} & =J_{n} \circ T^{n} .
\end{aligned}
$$

In particular Rota's dilation is

$$
J_{0} \circ T^{2 n}=\mathbb{E}_{0]} \circ \mathbb{E}_{[n} \circ J_{0} .
$$

The above construction can also be carried out for Fourier multipliers on discrete groups. Let $G$ be a discrete countable group and $L(G) \subset \mathbb{B}\left(\ell_{2} G\right)$ its left von Neumann algebra. It is the bicommutant of the left translations by $g \in G$, denoted as usual by $\lambda(g)$. It is a type $I I_{1}$ algebra with trace given by

$$
\tau(\lambda(g))=\left\langle\delta_{e}, \lambda(g) \delta_{e}\right\rangle=\delta_{g, e} .
$$

A function $t: G \rightarrow \mathbb{C}$ defines a Fourier multiplier $M_{t}$ if the following map is well defined on $L(G)$ :

$$
M_{t}(\lambda(g))=t_{g} \lambda(g) .
$$

Actually a Fourier multiplier is completely bounded if and only if the Schur multiplier $\left(t_{h^{-1} g}\right)_{h, g}$ is bounded on $\mathbb{B}\left(\ell_{2} G\right)$ (see [7). It is unital completely positive iff $t_{e}=1$ ( $e$ is the unit of $G$ ) and $t$ is positive definite; in this case it is $\tau$-Markovian. $M_{t}$ is self-adjoint if $t_{g}=t_{g^{-1}} \in \mathbb{R}$.

Corollary. Any self-adjoint unital completely positive Fourier multiplier on a discrete group is factorizable. 
Proof. The dilation can be obtained directly from the previous one but can be reinterpreted in terms of a cross product as follows.

Let $\ell_{2, T}$ be the Hilbert space obtained by quotient and completion of the real span of $\lambda(g)$ for the scalar product

$$
\langle\lambda(g), \lambda(h)\rangle_{T}=t_{g^{-1} h}
$$

We let $h$ be the class of $\lambda(h)$ in $\ell_{2, T}$. It is clear that $G$ acts unitarily on $\ell_{2, T}$ by left multiplications. So $G$ also acts by automorphisms on $\Gamma_{-1}\left(\ell_{2, T}\right)$ by, for $g, h \in G$,

$$
\alpha(g) \cdot \omega(h)=\omega(g h) .
$$

Let $\tilde{M}$ be the crossed product $\Gamma_{-1}\left(\ell_{2, T}\right) \rtimes_{\alpha} G$ (see [13] for definitions). This is again a type $I I_{1}$ algebra, $\tilde{\phi}$ is the canonical trace and for $g \in G, x \in \ell_{2, T}$,

$$
\lambda(g) \omega(x) \lambda\left(g^{-1}\right)=\alpha(g) \cdot \omega(x)=\omega(g \cdot x) .
$$

Then $\pi$ is just the natural copy of $L(G)$ in $\tilde{M}$ and $\rho$ is given by $\rho(x)=$ $\omega(e) \pi(x) \omega(e)$ for $x \in L(G)$. Note that $\omega(e)$ is a symmetry. We have

$$
\begin{aligned}
\tilde{\phi}(\pi(\lambda(g)) \rho(\lambda(h))) & =\tilde{\phi}(\lambda(g) \omega(e) \lambda(h) \omega(e)) \\
& =\tilde{\phi}\left(\lambda(g) \lambda(h) \omega\left(h^{-1}\right) \omega(e)\right) \\
& =\delta_{g h, e} t_{h}=\delta_{g h, e} t_{g} \\
& =\tau\left(M_{t}(\lambda(g)) \lambda(h)\right) .
\end{aligned}
$$

As before, one has nice formulas for the associated Markov chain. Briefly speaking, $M=\Gamma_{-1}\left(\ell_{2, T} \otimes \ell_{2}\right) \rtimes_{\alpha} G$, where $\alpha$ is the diagonal action of $G: \alpha(g) \cdot \omega(h \otimes v)=$ $\omega(g h \otimes v)$ for $g, h \in G$ and $v \in \ell_{2}$. The inclusion $J_{0}$ of $L(G)$ is the natural one. The morphism $\beta$ is given by the shift on the canonical basis $\left(e_{i}\right)$ of $\ell_{2}$ and a conjugation

$$
\beta\left(\lambda(g) \omega\left(h \otimes e_{i}\right)\right)=\omega\left(e \otimes e_{0}\right) \lambda(g) \omega\left(h \otimes e_{i+1}\right) \omega\left(e \otimes e_{0}\right) .
$$

Then one defines $\mathcal{B}_{n]}$ and $\mathcal{B}_{[n}$ as above to get Rota's construction.

There is another family of maps close to multipliers which can be seen to have a dilation quite easily. It consists of the maps arising from the second quantization on $q$-deformed algebras (see [2]). For any real Hilbert space $K$, let $\omega_{K}: \Gamma_{q}(K) \rightarrow$ $\mathcal{F}_{q}(K)$ be $\omega_{K}(x)=x \cdot \Omega_{K}$. This is an injective mapping with dense range; we denote by $\omega_{K}^{-1}$ its inverse (not defined everywhere).

If $K$ and $L$ are real Hilbert spaces, any contraction $T: K \rightarrow L$ gives rise to a tracial Markov map $\Gamma(T): \Gamma_{q}(K) \rightarrow \Gamma_{q}(L)$ satisfying

$$
T\left(\omega_{K}^{-1}\left(k_{1} \otimes \ldots \otimes k_{n}\right)\right)=\omega_{L}^{-1}\left(T\left(k_{1}\right) \otimes \ldots \otimes T\left(k_{n}\right)\right) .
$$

If $T$ is isometric (unitary), then $\Gamma(T)$ is an injective representation (automorphism). So if $K \subset L$, we can see $\Gamma_{q}(K)$ as a subalgebra of $\Gamma_{q}(L)$. In this situation, if $P$ is the orthogonal projection of $L$ onto $K, \Gamma(P)$ is then the trace-preserving conditional expectation from $\Gamma_{q}(L)$ onto $\Gamma_{q}(K)$.

Any contraction $T: K \rightarrow K$ can be dilated to a unitary say $U$ (to a symmetry if $T$ is self-adjoint) on a bigger Hilbert space $L$. From $T=P U_{\mid K}$, one sees that a dilation of $\Gamma(T)$ is given by $\Gamma_{q}(L)$ with its trace, with $\pi$ the natural injection and $\rho=\Gamma\left(U^{*}\right) \circ \pi$.

To get Rota's dilation is also easy in this case, assuming that $T$ is self-adjoint. Indeed, let $U$ be a strong dilation of $T$ on $L$, that is, $P U_{\mid K}^{k}=T^{k}$ (see [7, Theorem 1.1). Let $K_{n}=\operatorname{span}\left\{U^{l}(K) ; l \geqslant n\right\}$ and $P_{n}$ be the corresponding projection. 
Then one has

$$
P P_{n} P=T^{2 n}
$$

Indeed if $k \in K$ and $x \in K$ for $l \geqslant 0$,

$$
\begin{aligned}
\left\langle k, U^{n+l}(x)\right\rangle & =\left\langle k, P U^{n+l} P(x)\right\rangle=\left\langle T^{n+l}(k), x\right\rangle \\
& =\left\langle T^{n}(k), U^{l}(x)\right\rangle=\left\langle U^{n}\left(T^{n}(k)\right), U^{n+l}(x)\right\rangle,
\end{aligned}
$$

so $P_{n} P(k)=U^{n}\left(T^{n}(x)\right)$.

Going to the second quantization, with $\mathbb{E}_{n}=\Gamma\left(P_{n}\right)$ being the conditional expectation from $\Gamma_{q}(L)$ onto $\Gamma_{q}\left(K_{n}\right)$, and $\hat{\mathbb{E}}=\Gamma(P)$ being the conditional expectation onto $\Gamma_{q}(K)$ (adjoint of the inclusion $J$ ), one has

$$
\hat{\mathbb{E}} \circ \mathbb{E}_{n} \circ J=\Gamma\left(P P_{n} P\right) \circ J=J \circ \Gamma(T)^{2 n} .
$$

The same dilation works also for the $q$-deformed versions of the Araki-Woods factors of Hiai ([3]).

\section{REFERENCES}

[1] Claire Anantharaman-Delaroche, On ergodic theorems for free group actions on noncommutative spaces, Probab. Theory Related Fields 135(4) (2006), 520-546. MR2240699 (2007m:46104)

[2] Marek Bożejko, Burkhard Kümmerer, and Roland Speicher, q-Gaussian processes: Noncommutative and classical aspects, Comm. Math. Phys. 185(1) (1997), 129-154. MR 1463036 (98h:81053)

[3] Fumio Hiai, q-deformed Araki-Woods algebras, Operator algebras and mathematical physics (Constanţa, 2001), Theta, Bucharest, 2003, pp. 169-202. MR2018229 (2004j:46086)

[4] M. Junge, Operator spaces and Araki-Woods factors: A quantum probabilistic approach, IMRP Int. Math. Res. Pap. 87 (2006), Art. ID 76978. MR2268491

[5] Marius Junge, Christian Le Merdy, and Quanhua $\mathrm{Xu}, H^{\infty}$ functional calculus and square functions on noncommutative $L^{p}$-spaces, Astérisque, vol. 305, 2006. MR 2265255

[6] Marius Junge and Quanhua Xu, Noncommutative maximal ergodic theorems, J. Amer. Math. Soc. 20(2) (2007), 385-439. MR2276775 (2007k:46109)

[7] Gilles Pisier, Similarity problems and completely bounded maps, Lecture Notes in Math., vol. 1618, Springer-Verlag, Berlin, 2001. MR1818047 (2001m:47002)

[8] Yves Raynaud, On ultrapowers of non commutative $L_{p}$ spaces, J. Operator Theory 48(1) (2002), 41-68. MR1926043 (2003i:46069)

[9] Éric Ricard and Quanhua Xu, Khintchine type inequalities for reduced free products and applications, J. Reine Angew. Math. 599 (2006), 27-59. MR:2279097

[10] Gian-Carlo Rota, An "Alternierende Verfahren" for general positive operators, Bull. Amer. Math. Soc. 68 (1962), 95-102. MR0133847 (24:A3671)

[11] Jean-Luc Sauvageot, Markov quantum semigroups admit covariant Markov $C^{*}$-dilations, Comm. Math. Phys. 106(1) (1986), 91-103. MR853979 (88a:46077)

[12] Elias M. Stein, Topics in harmonic analysis related to the Littlewood-Paley theory, Annals of Mathematics Studies, No. 63, Princeton University Press, Princeton, NJ, 1970. MR0252961 (40:6176)

[13] M. Takesaki, Theory of operator algebras. II, volume 125 of Encyclopaedia of Mathematical Sciences, Springer-Verlag, Berlin, 2003. MR1943006 (2004g:46079)

[14] D. V. Voiculescu, K. J. Dykema, and A. Nica, Free random variables, volume 1 of CRM Monograph Series, American Mathematical Society, Providence, RI, 1992. MR1217253(94c:46133)

Laboratoire de Mathématiques, Université de Franche-Comté, 25030 Besançon, CEDEX, FRANCE

E-mail address: eric.ricard@univ-fcomte.fr 